\title{
Diseño y análisis de la violencia de género aplicando la técnica de respuesta aleatoria
}

\author{
Design and analysis of gender-based violence using the random response \\ technique
}

Carmen Estrella Morocho Barrionuevo. ${ }^{1}$, Tania Paulina Morocho Barrionuevo. ${ }^{2} \&$ Diana Katherine Campoverde Santos. ${ }^{3}$

Recibido: 24-01-2021 / Revisado: 29-01-2021 /Aceptado: 24-02-2021/ Publicado: 05-03-2021

\begin{abstract}
. DOI: https://doi.org/10.33262/concienciadigital.v4i1.2.1602

Introduction. Warner (1965) was a pioneer in establishing the random response technique to reduce alterations of the estimators due to false or conditioned responses. From this contribution there were later techniques such as the unrelated response technique proposed by Greenberg (1969) and the random response technique by Liu Chow (1976). Objective. Compare the random response techniques with the direct response technique, to estimate the true proportion of the sensitive characteristics in the study of gender violence while protecting the privacy of the respondents since by requiring direct responses this information may be altered. Methodology. The samples were established for each technique, thus having a total of 998 out of a population of 2205 students from Universidad Nacional de Chimborazo, the questionnaire was composed of 15 items. Results. The random response techniques reveal an increase compared to the direct response technique, according to the estimates and confidence intervals obtained in the results. Conclution. Ultimately, thanks to the study carried out, it is found that the random response technique is more effective than the direct response technique, because

\footnotetext{
${ }^{1}$ Universidad Nacional de Chimborazo, Riobamba, Ecuador, carmen.morocho@unach.edu.ec.

${ }^{2}$ Universidad de Granada, Escuela Internacional de Postgrado. Granada,

España.taniapaulina@correo.ugr.es.

${ }^{3}$ Escuela Superior Politécnica de Chimborazo, Agroindustria, Riobamba, Ecuador. diana.campoverde@espoch.edu.ec, https://orcid.org/0000-0001-8538-6747.
} 
it provides better estimators in all sensitive questions, since the questions are the same in both surveys. Also, it was confirmed that the respondents are more reluctant to give their answer through the direct response because perhaps the respondents think that their privacy is not well protected with this procedure. This is not the case in the Greenberg and Liu - Chow technique.

Keywords: Gender violence, random response technique, Greenberg technique, Liu Chow technique, unrelated question. sensitive question.

\section{Resumen.}

Introducción. Warner (1965) fue pionero en establecer la técnica de respuesta aleatoria para reducir alteraciones de los estimadores por respuestas falsas o condicionadas. A partir de este aporte hubo técnicas posteriores como la técnica de respuesta no relacionada propuesta por Greenberg (1969) y la técnica de respuesta aleatoria de Liu-Chow (1976). Objetivo. Comparar las técnicas de respuesta aleatoria con la técnica de respuesta directa, para estimar la verdadera proporción de las características sensibles en el estudio de violencia de género mientras se protege la privacidad de los encuestados ya que al requerir respuestas directas esta información puede verse alterada. Metodología. Las muestras fueron establecidas para cada técnica, teniendo así un total 998 de una población de 2205 alumnos de la Universidad Nacional de Chimborazo, el cuestionario estuvo compuesto de 15 ítems. Resultados. Las técnicas de respuestas aleatorias revelan un incremento comparando con la técnica de respuesta directa, de acuerdo con las estimaciones e intervalos de confianza obtenidos en los resultados. Conclusión. En definitiva, gracias al estudio realizado se comprueba que es más eficaz la técnica de respuesta aleatoria que la técnica de respuesta directa, porque proporciona mejores estimadores en todas las preguntas sensibles, siendo que en ambas encuestas las preguntas son iguales. También, se pudo corroborar que los encuestados son más reacios a dar su respuesta a través de la respuesta directa porque tal vez los encuestados piensan que su privacidad no está bien protegida con este procedimiento. Este no es el caso en la técnica de Greenberg y LiuChow.

Palabras claves: Violencia de género, técnica de respuesta aleatoria, técnica Greenberg, técnica de Liu Chow, pregunta no relacionada. pregunta sensitiva.

\section{Introducción.}

En este artículo analizaremos la violencia de género contra las mujeres en la Universidad Nacional de Chimborazo empleando la técnica de respuesta aleatorizada, en estudios anteriores se encontró que las mujeres han sido víctimas de violencia en general presentando afectación emocional. (Chávez \& Juárez, 2016)

Los investigadores enfrentan dificultades en la búsqueda de las respuestas seguras, en 
investigaciones sociales sobre aspectos sensibles como la drogadicción, comportamiento sexual, violencia doméstica, prácticas delictivas, entre otros. Los encuestados tienden a responder en función de lo que creen que deberían responder, en ocasiones ocultan información brindando respuestas falsas o no responden, es así la importancia de utilizar las técnicas de respuesta aleatorizada (RR) cuyo objetivo principal es recopilar información fiable protegiendo la confidencialidad del encuestado evitando la no respuesta.

Warner (1965) propuso la Técnica de Respuesta Aleatorizada que permite obtener información sobre una característica sensible, garantizando la protección del anonimato del entrevistado, reduciendo la no respuesta o evasión de preguntas sensibles. Radica en el manejo de un dispositivo aleatorio tomando dos preguntas: ¿pertenece al equipo de la característica sensible? o ¿no pertenece al equipo de la característica sensible? El entrevistado podrá responder "Sí" o "No", sin embargo, el entrevistador no tendrá la opción de conocer que pregunta contestó el entrevistado, resguardando la confidencialidad de este.

Además, en los últimos años se ha considerado como un problema de salud pública lo cual se ha sido reconocido por la colectividad (Salud, 2013). Una de las investigaciones se desarrolló en América Latina y el Caribe utilizando encuestas Demográficas y de Salud (DHS), y Encuestas de Salud Reproductiva (RHS), recogidas entre los años 2003 - 2009 en 12 países, entre ellos Ecuador. La entrevista cara a cara fue el medio de recopilación de información, en el análisis de la información sobre "la aceptabilidad que al menos por una razón el marido golpee a la esposa", es de 38,2\% en Ecuador 2004. Mayor significación se encuentra entre mujeres del sector rural que mujeres urbanas, y en mujeres que habían tolerado física o sexual por su pareja en los doce meses anteriores, en comparación con las mujeres que no la habían sufrido ninguna violencia. (Bottalessandra, 2013).

En el estudio denominado "Underreporting of Gender-Based Violence in Kerala, India An Aplication of the List Randomization Method", donde se analizó el grado de incidencia de violencia doméstica y acoso físico en público, utilizaron la Técnica de lista aleatoria, indicando que el nivel de violencia doméstica supera el $9 \%$ y despreciable para problemas físicos, de acoso en autobuses públicos/ privados. (Joseph, y otros, 2017)

En Ecuador desde el año 1980 se firma la Convención para la Exclusión de la Discriminación en todas las Formas para la Mujer, en 1994 se crearon las Comisarías de la Mujer y la Familia (CMF), instancias especializadas en la administración de justicia de atención integral a las usuarias, enfocado a prevenir, atender, juzgar y sancionar la violencia intrafamiliar. (Camacho, 2014) En el año 2011 se realizó la primera Encuesta Nacional sobre Relaciones Familiares y Violencia de Género contra las mujeres por el Instituto Nacional de Estadísticas y Censos, como resultado se obtuvo, 6 de cada 10 mujeres reconocen haber sufrido malos tratos. El 70,5\% de mujeres que se han unido o que han contraído matrimonio entre los 16 y 20 años son las que mayor violencia han 
vivido. (Censos, Encuesta Nacional de Relaciones Familiares y Violencia de Género contra las Mujeres, 2012).

Para el año 2018 en el Registro Oficial N 175 se aprueba la Ley Orgánica Integral para la prevención y eliminación de la Violencia Contra las Mujeres, cuyos objetivos buscan enfocar la acción del Estado en sensibilizar y prevenir de la violencia con la colaboración de la ciudadanía, además, de considerar tres mecanismos para la eliminación de la violencia: atender, proteger y reparar a las víctimas de violencia garantizando su integralidad y seguridad con la finalidad que retomen su vida. (Nacional, 2018) En el año 2019 se realiza una segunda Encuesta, a nivel nacional, para actualizar la información en la prevalencia de la violencia en sus distintas formas: psicológica, física, sexual y económica que sufrieron las mujeres mayores o iguales a 15 años en los diferentes ámbitos públicos como privados, e identificar a sus agresores y el lugar en que sucedieron los hechos de violencia. (Hidalgo N., Aguirre C., Luna I., Suasnavas A. and Albán A, 2019)

En Ecuador, el Instituto Nacional de Estadísticas y Censos, realizó un estudio para analizar la violencia de género, donde aplicaron muestreo probabilístico, cuya población objetivo fueron mujeres de 15 años a más, con un periodo de referencia de: "a lo largo de la vida" y "En los últimos 12 meses", obteniendo como resultados que 65 de cada 100 mujeres experimentaron un acto de violencia en su vida, 32 de cada 100 mujeres han experimentado un acto de violencia en los en los 12 últimos meses, de las cuales el $40.8 \%$ son mujeres entre 18 a 29 años. (Censos, Encuesta Nacional sobre Relaciones Familiares y Violencia de Género contra las Mujeres -ENVIGMU, 2019)

El objetivo de este trabajo es determinar el diseño y análisis de una encuesta para el estudio de la violencia de género en estudiantes de la Facultad de Ciencias Políticas y Administrativas de la Universidad Nacional de Chimborazo, Ecuador, utilizando la Técnica de Respuesta Aleatorizada Liu Chow (RA) y la Técnica de la pregunta no relacionada (RN) desarrollada por Bernard G. Greenberg. La violencia de género es una problemática que ocurre en toda clase social y ámbitos de la Sociedad, se considera todo acto de violencia o agresión, basado en una situación de desigualdad. Según la Organización de Naciones Unidas (ONU), es todo acto de violencia al sexo femenino, obteniendo como resultado un perjuicio o sufrimiento psicológico, sexual, físico, así como también amenazas, la ausencia arbitraria de la libertad, ocurriendo en la vida privada o pública (O.N.U., 1993).

\section{Metodología.}

\section{A. Técnicas de Respuesta Aleatorizada}

La técnica de Respuesta Aleatorizada (RR), introducida en 1965 por Stanley Warner, ha sido diseñada para minimizar la no respuesta sobre aspectos sensibles y para salvaguardar la confidencialidad de la persona entrevistada, (Warner, 1965), es eficaz para reducir los 
errores ajenos al muestreo y revelar el estado real de los encuestados, como por ejemplo: la negativa a responder o a su vez mentir en encuestas, son 2 razones principales de tales errores ajenos al muestreo.

Warney (Warner, 1965) propone el método W como una técnica que clasifica a las personas en grupos. Cada persona va a estar en uno de los grupos, de tal manera que la proporción será conformada por individuos con ciertas características de interés. El modelo se basa en determinar si una persona pertenece directamente o no a un grupo. La técnica RR ha sido determinada por Warner aplicable al esquema de muestreo aleatorio simple con reemplazamiento. El método genérico se basa en extraer una muestra aleatoria de tamaño $n$ según un diseño de muestreo seleccionado como: aleatorio simple, estratificado, conglomerados, probabilidades proporcionales a los tamaños, entre otros. Cada elemento de la muestra o entrevistado, seleccionará una de las dos preguntas complementarias, y responderá $\mathrm{V}$ verdadero o $\mathrm{F}$ si es falso. Mediante el modelo estadístico W se estima el total de personas con la característica sensible.

Para lograr que el entrevistado tenga mayor disposición a cooperar con la encuesta, (Greenberg B., Lafif A., Abul-Ela A., Walt R., Daniel G., and Horvitz D, 1969) modificó el modelo de Warner, generándose de esta forma el modelo U. Su desarrollo es semejante al modelo $\mathrm{W}$, tiene un mecanismo aleatorio que selecciona una de las 2 preguntas, no obstante, una pregunta corresponderá al aspecto sensible y la segunda pregunta no va a estar relacionada, lo que producirá una contestación afirmativa con probabilidad $\alpha$ conocida, que generalmente la pregunta que responde el aspecto sensible tiene una probabilidad $p$, y la no relacionada $1-p$. Analizados los modelos $\mathrm{W}$ y $\mathrm{U}$ en un marco de poblaciones finitas por (Moors, 1971), el modelo W es el más eficiente.

Además, Soberanis et al. (2008) presentan el modelo C manteniendo la confidencialidad del entrevistado y plantean que, para mejorar la exactitud de un estimador, se debería incorporar información auxiliar correlacionada con la variable de interés, es decir, relacionando una pregunta inocua del modelo U y la variable sensitiva. (Soberanis, 2008)

Así mismo Hortvitz et al. (1976) realizan el modelo H, el cual posibilita que el encuestado reciba una mayor protección de anonimato, sin que se requiera una pregunta complementaria. Para ello cada elemento de la muestra, selecciona al azar una de tres proposiciones: (1) la pregunta sensible, (2) una instrucción que dice sí y (3) una instrucción que dice no, con probabilidades $p_{1}, p_{2}$ y $p_{3}$, donde $p_{1}+p_{2}+p_{3}=1$. (Horvitz D., Shah B., and Simmons, W, 1967)

Puede agregarse que un año más tarde Devore (Devore J, 1977), sugiere un modelo D, parecido al modelo $\mathrm{U}$, no obstante, la pertenencia al grupo inocuo $\mathrm{W}$ se define con probabilidad uno.

Añádase a estos modelos el modelo M, propuesto por Mangat y Singh (Mangat, 1990), es una técnica de respuesta aleatorizada equivalente a la técnica de Warner, su mecanismo 
aleatorio proporciona $n$ respuestas independientes con 2 componentes aleatorios. Dos proposiciones conforman la primera componente, las cuales son seleccionadas con probabilidades T y $1-\mathrm{T}$. A la persona muestreada $i$ se le presentan dos cajas, se le solicita a la persona que escoja aleatoriamente una carta de una serie de cartas marcadas $\mathrm{T}$ o $\mathrm{R}$ en proporción $T$ : $(1-T),(0<T<1)$. Se escoge una carta, se observa y se devuelve a la caja. Si la carta está marcada $\mathrm{T}$, la persona debe informar el valor verdadero $y_{i}$, el cuál es 1 si está marcada con A y 0 si i está marcada con $A^{c}$. Si la carta está marcada con $\mathrm{R}$ escogerá de la segunda caja que contiene cartas marcadas con $A$ y $A^{c}$ en proporciones p: $(1-p),(0<p \neq 1 / 2<1)$. La respuesta aleatoria de la persona i se asume que es: $I_{i}=1$ la carta coincide con la característica verdadera $A / A^{c}, I_{i}=0$ la carta no coincide. Mientras que, la respuesta aleatoria de i puede denotarse como $z_{i}:=y_{i}$ si la primera caja la carta es $\mathrm{T}, z_{i}=I_{i}$ si la primera caja la carta es $\mathrm{R}$.

\section{B. Técnica de Respuesta Aleatorizada Liu Chow}

En circunstancias en las que se buscan respuestas potencialmente delicadas o incriminatorias, la técnica de RR, propuesta inicialmente por Warner (1965), es eficaz en la disminución de errores no muestrales en encuestas por muestreo. Generalmente a los encuestados al impartirles preguntas de carácter delicado (por ejemplo: uso de drogas, comportamiento sexual, entre otros) tienden a negarse a responder o mentir, determinando errores no muestrales, por prejuicios de que pueda conducir a una discriminación.

Al principio, el RR fue diseñado para manejar respuestas binomiales (sí / no) a preguntas sensibles como ¿Alguna vez ha consumido drogas ilegales? El modelo inicial suponía proporcionan dos preguntas sí / no para cada encuestado, y que se usó un dispositivo de aleatorización para determinar qué la pregunta sería respondida. Considerando que el entrevistador no sabría el resultado del mecanismo de aleatorización, los participantes serían alentados a ofrecer información veraz a la pregunta sensible. Desde la publicación del Modelo de Respuesta Aleatoria de Warner en 1965, muchos autores han estudiado su modelo principal. (Kim, 2005)

Entre estas tenemos (Antonak R. and Lvneh H., 1995), (Chaudhuri A. and Mukerjee R., 1988), (Fox J. and Tracy P., 1986), (Scheers N, 1992) y (Umesh U. and Peterson R., 1991), (Abdul-Ela A., Horvitz D. and Greenberg B., 1967), en las que se generalizó la RR dicotómica de Warner a un entorno multinomial para incluir respuestas multivariadas a preguntas como ¿Cuántos días consumió drogas ilegales la semana pasada? Por ejemplo, las posibles respuestas a esta pregunta pueden ser; $0,1,2 \mathrm{o} \geq 3$, en cuyo caso un modelo multinomial puede ser apropiado. Esta extensión indujo otros autores para considerar el RR para respuestas cuantitativas como la técnica propuesta por (Liu P. and Chow L., 1976). Estos autores propusieron un modelo de respuesta cuantitativa discreta utilizando el dispositivo de aleatorización de Hopkins.

El dispositivo de aleatorización usado en el modelo propuesto por Liu Chow, tiene una serie de bolas de 2 colores diferentes rojo y blanco, las mismas que se colocarán dentro 
del dispositivo. Un número discreto, como $0,1,2, \ldots, \mathrm{k}$ se marcará en la superficie de cada una de las bolas blancas. La proporción de bolas rojas, y de bolas blancas con diferentes figuras $(0,1,2, \ldots, \mathrm{k})$ estará predeterminado.

Se pide al encuestado que seleccione una bola con el dispositivo. Si es una bola roja, se le pedirá al encuestado que responda la pregunta delicada (por ejemplo, el número de abortos que ella ha tenido). Si la bola es blanca, habrá un número marcado en su superficie, y el encuestado simplemente dice el número. Las respuestas nuevamente serán $0,1,2, \ldots, \mathrm{k}$, dependiendo del número marcado en superficie de la bola blanca. El entrevistador desconoce el color que apareció en la bola, por lo tanto, no sabe si el encuestado ha contestado a la pregunta sensible o no.

\section{Estimación de parámetros}

Sea $\mathrm{w}=$ número de bolas color blanco marcadas con $\mathrm{i}$ (donde $\mathrm{i}=0,1,2, \ldots, \mathrm{k}), r=$ número de bolas color rojo (sin marcar), luego el número total de bolas en el dispositivo es $r+$ $\sum_{i=0}^{k} w_{i}=r+w\left(\right.$ donde $\left.w=\sum_{i=0}^{k} w_{i}\right)$. Si $\pi_{i}$ representa la verdadera proporción de encuestados que poseen el valor $i$ en la medida cuantitativa (donde $\sum_{i=0}^{k} \pi_{i}=1$ ), entonces la probabilidad de que un encuestado seleccionado al azar de una población responda "i" es:

$$
P_{i}=\pi_{i}\left(\frac{r}{(r+w)}\right)+\left(\frac{w_{i}}{(r+w)}\right)
$$

Si $\mathrm{n}=$ tamaño de la muestra aleatoria, y $n_{i}=$ número de personas que responden a la pregunta ( $i$ ), una estimación de $P_{i}$ viene dada por:

$$
\hat{\pi}_{i}=\frac{(r+w) Y_{i}}{r}-\frac{w_{i}}{r}
$$

La varianza para $\hat{\pi}_{i}$, y la covarianza para $\hat{\pi}_{i}, \hat{\pi}_{j}$ son:

$$
\begin{aligned}
& \widehat{\operatorname{Var}}\left(\hat{\pi}_{i}\right)=\left(\frac{r+w}{r}\right)^{2} \frac{\left(Y_{i}\left(1-Y_{i}\right)\right)}{n} \\
& \widehat{\operatorname{Cov}}\left(\hat{\pi}_{i}, \hat{\pi}_{j}\right)=-\left(\frac{r+w}{r}\right)^{2} \frac{Y_{i} Y_{j}}{n}
\end{aligned}
$$

La ecuación (3) muestra que la proporción de bolas rojas en el dispositivo es el principal componente que afecta la eficiencia de la estimación. Si el número total de bolas es fijo y el número de bolas rojas aumenta, entonces aumentará la eficiencia de la estimación. Si el número total de bolas aumenta, pero el número de bolas rojas también aumentan proporcionalmente (proporción de bolas rojas al número total de bolas sin cambios), entonces la eficiencia de la estimación no ha cambiado. Si la proporción de bolas rojas para el número total de bolas se acerca a uno (es decir, el dispositivo solo contiene las bolas rojas), entonces el modelo es idéntico al método convencional de preguntas directas. 
El método actual es una modificación del modelo cuantitativo de Greenberg, utilizando una distribución predeterminada de atributos no sensibles (representados por un número de bolas blancas con marcas de diferentes figuras) en lugar de una pregunta inocua. Debido a esta modificación, la eficiencia de la estimación debe mejorarse importantemente. Es interesante notar que la eficiencia de la estimación con este nuevo modelo depende únicamente de la relación entre la cantidad total de bolas y la cantidad de bolas rojas $((\mathrm{r}+\mathrm{w}) / \mathrm{r})$. Cambiar el número total de bolas en el dispositivo no afectará la eficiencia. Con una relación fija de $((\mathrm{r}+\mathrm{w}) / \mathrm{r})$, puede ser posible aumentar la respuesta de un encuestado aumentando el número total de bolas o cambiar la proporción de bolas blancas. Incrementar el número de ensayos por encuestado debe nuevamente mejorar la eficiencia de la estimación, pero al costo de mayor sospecha de los encuestados.

\section{Técnica de la pregunta no relacionada desarrollada por Bernard G. Greenberg}

Los artículos publicados en los años de 1967 y 1969 por Horvitz et al. y Greenberg et al. respectivamente presentan una modificación a la técnica de respuesta aleatoria de Warner (1965). En esta técnica se amplió y perfeccionó la investigación realizada por Warner, llamada pregunta no relacionada, para la medición de variables cualitativas, y cuantitativas.

El método aplicable para variables cualitativas es un procedimiento alternativo al desarrollado por Warner en el cual se espera incrementar la respuesta del encuestado, considerando una segunda pregunta no relacionada al tema sensible, con respecto a la primera. Por ejemplo, se esperaría aseverar o negar "Nací el mes de agosto", comparado con la pregunta sensible "Pertenezco al atributo A", siendo la clase "A" el grupo con la característica sensible que se estudia.

Sí todos los encuestados contestan con la verdad, la proporción de las respuestas afirmativas se convierte en $\emptyset=P \pi_{A}+(1-p)\left(\pi_{N R}\right)$, donde NR significa no relacionada y $\pi_{N R}$ es la proporción de la población encuestada que nacieron en el mes de agosto. Si conocemos el valor de $\pi_{N R}$, el valor estimado de $\hat{\pi}_{N R}=\frac{\left[\widehat{\varnothing}-(1-p) \pi_{N R}\right]}{P}$ y la varianza es $V\left(\hat{\pi}_{A N R}\right)=\frac{\emptyset(1-\emptyset)}{n P^{2}}$.

De otro lado en el modelo cuantitativo al utilizar 2 preguntas, la distribución de los resultados está relacionada a respuestas numéricas, por lo cual las respuestas de la variable inocua tienen que estar en el mismo rango que las de la variable original.

Como se puede observar la distribución es una mezcla de dos distribuciones individuales, las cuales deben estar separadas estadísticamente para conseguir estimadores de los parámetros de investigación que sean significativos. La media poblacional de las distribuciones sensibles y no sensibles se define como $\mu_{A}$ y $\mu_{\gamma}$ y sus varianzas $\sigma_{A}^{2}$ y $\sigma_{\gamma}^{2}$.

Suponiendo que las dos muestras son independientes de tamaño $n_{1}$ y $n_{2}$, y no tienen repeticiones, establecemos que: 
- $\quad p_{i}=$ probabilidad de ser seleccionada la pregunta sensible por el encuestado en la muestra $i(i=1,2)$, donde $p_{1} \neq p_{2}$.

- $1-p_{i}=$ probabilidad de ser seleccionada la pregunta no sensible por el encuestado en la muestra $i(i=1,2)$.

- $Z_{i j}=$ respuesta de la j-ésima persona en la muestra $i(i=1,2), j=\left(1,2, \ldots \ldots, n_{i}\right)$

- $f(z)=$ función de probabilidad asociada con la pregunta sensible, $E_{f}[Z]=\mu_{A}$.

- $g(z)=$ función de densidad de probabilidad asociada con la pregunta no sensible (similar a $f(z)$ en el rango de respuesta), con $E_{g}[Z]=\mu_{\gamma}$.

- $\hat{\mu}_{A}=$ estimador muestral de la media de la distribución sensible.

- $\hat{\mu}_{\gamma}=$ estimador muestral de la media de la distribución no sensible.

La función de probabilidad para cada elemento en una muestra es:

$$
\begin{array}{cc} 
& \text { Muestra } 1: \Psi_{1}\left(z_{1}\right)=p_{1} f\left(z_{1}\right)+\left(1-p_{1}\right) g\left(z_{1}\right) \\
& \text { Muestra 2: } \Psi_{2}\left(z_{2}\right)=p_{2} f\left(z_{2}\right)+\left(1-p_{2}\right) g\left(z_{2}\right) \\
\text { Entonces: } & \mu_{z_{1}}=E\left[Z_{1}\right]=p_{1} \mu_{A}+\left(1-p_{1}\right) \mu_{\gamma} \\
& \mu_{z_{2}}=E\left[Z_{2}\right]=p_{2} \mu_{A}+\left(1-p_{2}\right) \mu_{\gamma}
\end{array}
$$

Al remplazar $\mu_{z_{1}}$ y $\mu_{z_{2}}$, por las medias de las muestras $\overline{Z_{1}} y \overline{Z_{2}}$, respectivamente, teniendo como resultados estimadores insesgados para $\mu_{A}$ y $\mu_{\gamma}$ :

$$
\widehat{\mu_{A}}=\frac{\left(1-p_{2}\right) \overline{Z_{1}}-\left(1-p_{1}\right) \overline{Z_{2}}}{p_{1}-p_{2}} \quad \text { (5) } \quad \widehat{\mu_{\gamma}}=\frac{p_{2} \overline{Z_{1}}-p_{1} \overline{Z_{2}}}{p_{2}-p_{1}}
$$

Varianzas respectivas:

$$
\begin{aligned}
& V\left(\widehat{\mu_{A}}\right)=\frac{1}{\left(p_{1}-p_{2}\right)^{2}}\left[\left(1-p_{2}\right)^{2} V\left(\overline{Z_{1}}\right)+\left(1-p_{1}\right)^{2} V\left(\overline{Z_{2}}\right)\right] \\
& V\left(\widehat{\mu_{\gamma}}\right)=\frac{1}{\left(p_{2}-p_{1}\right)^{2}}\left[p_{2}^{2} V\left(\overline{Z_{1}}\right)+p_{1}^{2} V\left(\overline{Z_{2}}\right)\right]
\end{aligned}
$$

Dando:

$$
V\left(\bar{Z}_{l}\right)=\frac{1}{n_{i}}\left[\sigma_{\gamma}^{2}+p_{i}\left(\sigma_{A}^{2}-\sigma_{\gamma}^{2}\right)+p_{i}\left(1-p_{i}\right)\left(\mu_{A}-\mu_{\gamma}\right)^{2}\right] \quad \mathrm{i}=1,2
$$

Los estimadores de las ecuaciones 5 y 6 , son calculados simplemente de los datos de la muestra, y se utilizan medidas muestrales sin importar la naturaleza específica de $f(z)$ y $g(z)$. La varianza de la muestra $s_{1}^{2}$ en las ecuaciones 7 y $8: \hat{V}\left(Z_{1}\right)=\frac{\sigma_{1}^{2}}{n_{1}} ; \widehat{V}\left(Z_{2}\right)=\frac{\sigma_{2}^{2}}{n_{2}}$; El óptimo diseño para una encuesta de Respuestas Aleatorizadas usando preguntas cuantitativas requiere de una elección apropiada para $p_{1}$ y $p_{2}$, la selección inteligente de la pregunta no sensibles Y, y una asignación eficaz del total de la muestra $n_{1}$ y $n_{2}$.

\section{Selección de la característica cuantitativa no sensibles}

Consideremos como regla fundamental que la pregunta no sensible debe de ser planteada de tal manera que la cantidad de la respuesta sea igual a la de la pregunta sensible, por 
ejemplo: monedas, distancias o número de veces en que ocurre un evento. A partir de un punto de vista intuitivo se puede pensar que las funciones de densidad de las respuestas a ambas preguntas no tienen solapes y que el cálculo de sus parámetros es independiente.

Además, la clasificación no está hecha en base a la respuesta dada individualmente sino en grupos usando operaciones para estimar, como con las ecuaciones (7, 8 y 9), para cualquier valor de $\left(\sigma_{A}, \sigma_{\gamma}, p_{1}, p_{2}, n_{1}, n_{2}\right)$ dados. Las varianzas de los estimadores aumentan cuando $\left|\mu_{A}, \mu_{\gamma}\right|$, aumenta.

Una vez que se han elegido $p_{1}$ y $p_{2}$ de acuerdo con el criterio anteriormente descrito, los otros parámetros manipulables son $n_{1}, n_{2}, \mu_{\gamma} y \sigma_{\gamma}^{2}$. Existe opción para $\mu_{A} y \sigma_{A}^{2}$ ya que dependen de la naturaleza de la característica sensible que no se conoce. Para cualquier valor de $\left(n_{1}, n_{2}\right)$ las varianzas de los estimadores decrecen cuando decrece $\sigma_{\gamma}^{2}$ y $\left|\mu_{A}, \mu_{\gamma}\right|$. Por eso la importancia de elegir una pregunta no sensible no es cuando difiere de las respuestas de la sensibilidad, sino en que tan parecidas son las respuestas. Una opción inteligente seria elegir una pregunta no sensible de tal forma que $\mu_{\gamma}$ se acerque a $\mu_{A} \mathrm{y}$ tenga una varianza $\sigma_{\gamma}^{2}$ mínima. Sin embargo, si $\sigma_{\gamma}^{2}$ es considerable menor que $\sigma_{A}^{2}$, puede haber perdidas en la cooperación por parte de los encuestados. Cualquiera de las respuestas al final de la distribución de A serían respuestas de A y esto es evidente para los encuestados más sagaces, que posiblemente darán una respuesta falsa en lugar de dar una respuesta verdadera que pudiera decir que pregunta están contestando. Por esta razón se recomienda que $\sigma_{\gamma}^{2}$ sea al menos tan grande como $\sigma_{A}^{2}$ y que todo caiga en la manipulación de $n_{1}$ y $n_{2}$ para reducir la varianza.

\section{Asignación para $n_{1}$ y $n_{2}$}

La subdivisión optima del total de la muestra de dos grupos se puede basar en el principio de minimizar $V\left(\widehat{\mu_{A}}\right)$. Esto se logra tomando:

$$
\frac{n_{1}}{n_{2}}=\sqrt{\frac{\left(1-p_{2}\right)^{2} V\left(Z_{1}\right)}{\left(1-p_{1}\right)^{2} V\left(Z_{2}\right)}}=\sqrt{\frac{\left(1-p_{2}\right)^{2}\left[1+p_{1}\left(\varnothing_{1}^{2}-1\right)+p_{1}\left(1-p_{1}\right) \emptyset_{2}^{2}\right]}{\left(1-p_{1}\right)^{2}\left[1+p_{2}\left(\varnothing_{1}^{2}-1\right)+p_{2}\left(1-p_{1}\right) \emptyset_{2}^{2}\right]}}
$$

Donde $\emptyset_{1}=\frac{\sigma_{A}}{\sigma_{\gamma}}, \emptyset_{2}=\frac{\left(\mu_{A}-\mu_{\gamma}\right)}{\sigma_{\gamma}}$ los primeros valores se usan para calcular $\frac{n_{1}}{n_{2}}$. Dependemos de un punto de referencia que muchas veces nos da una aproximación aceptable para la ecuación 10 como:

$$
\frac{n_{1}}{n_{2}} \approx \frac{p_{1}}{p_{2}} \operatorname{con} p_{1}+p_{2}=1
$$

Esto se debe a que cuando se eligen $p_{1}$ y $p_{2}$ que satisfagan $p_{1}+p_{2}=1$. La ecuación 11 es exacta cuando $\sigma_{A}^{2}=\sigma_{\gamma}^{2}$, y una aproximación cercana cuando $\sigma_{A}^{2} y \sigma_{\gamma}^{2}$ son diferentes, pero razonablemente cercanas entre sí, como deberían de ser para una buena elección de la pregunta sensible. Ya que la determinación de $\frac{n_{1}}{n_{2}}$ requiere el uso de valores intuitivos 
de la ecuación 11 para los parámetros poblacionales $\emptyset_{1}$ y $\emptyset_{2}$, es razonable suponer que en general 11 puede dar una asignación muy cercana a la óptima.

\section{Aplicación de la técnica de respuesta aleatorizada}

Desarrollaremos un estudio aplicando la técnica de respuesta aleatorizada para variables con categorías ordenadas, realizando una comparación de la técnica directa (RD) con las técnicas RA y RN. Las mismas que se basan en datos reales tomados en la Universidad de Nacional de Chimborazo realizados a una muestra representativa de estudiantes de diferentes carreras de la Facultad de Ciencias Políticas y Administrativas.

\section{Diseño de la muestra}

La población objetivo está constituido por 2205 estudiantes matriculados en el semestre octubre 2019-marzo 2020, en la Universidad Nacional de Chimborazo.

Se aplico un muestreo aleatorio simple para las tres encuestas con la particularidad:

- Para la RD con una probabilidad del $95 \%$ con un error $<0.05$, teniendo así una muestra de 298 estudiantes.

- Para la técnica de RA se tuvo una muestra de 350 estudiantes con un error de $<0.045$.

- Para la técnica de RN se dividió a la muestra de 350 estudiantes en dos submuestras.

\section{Diseño de la encuesta}

Las encuestas se aplicaron utilizando la aplicación Google Forms. La invitación se envió mediante correo electrónico en la que se encontraba el hipervínculo de la encuesta y el objetivo. Las preguntas para las 3 encuestas son las mismas; sin embargo, para el método de respuesta aleatorizada se utilizará preguntas no relacionadas el tema sensible, con probabilidades conocidas.

Para el análisis de los resultados de las encuestas se ha utilizado el software SPSS y R. Para concluir, se compararán los resultados obtenidos en los tres estudios.

\section{Formato de la encuesta}

La encuesta estuvo constituida por 15 preguntas (tabla 1), dividas por dos secciones en la primera sección está conformada con variables sociodemográficas de la pregunta 1 a la 6. En la sección dos las variables han sido divididas en dos grupos, las variables pertenecientes al grupo 1 desde la pregunta 7 a la 10 han sido medidas utilizando una escala de Likert de 5 puntos desde: 1=Nunca, 2= Rara vez, 3= Algunas veces, $4=$ Muchas veces, $5=$ Constantemente siempre, sistemáticamente. Para las variables sensibles del 
grupo 2, desde la pregunta 12 a la 15 han sido medidas utilizando una escala de Likert de 6 puntos: $1=$ Nunca, $2=1 \mathrm{vez}, 3=2-3$ veces, $4=4-10$ veces, $5=$ Más de 10 veces, $6=$ Prácticamente todos los días/ siempre.

Tabla 1. Preguntas

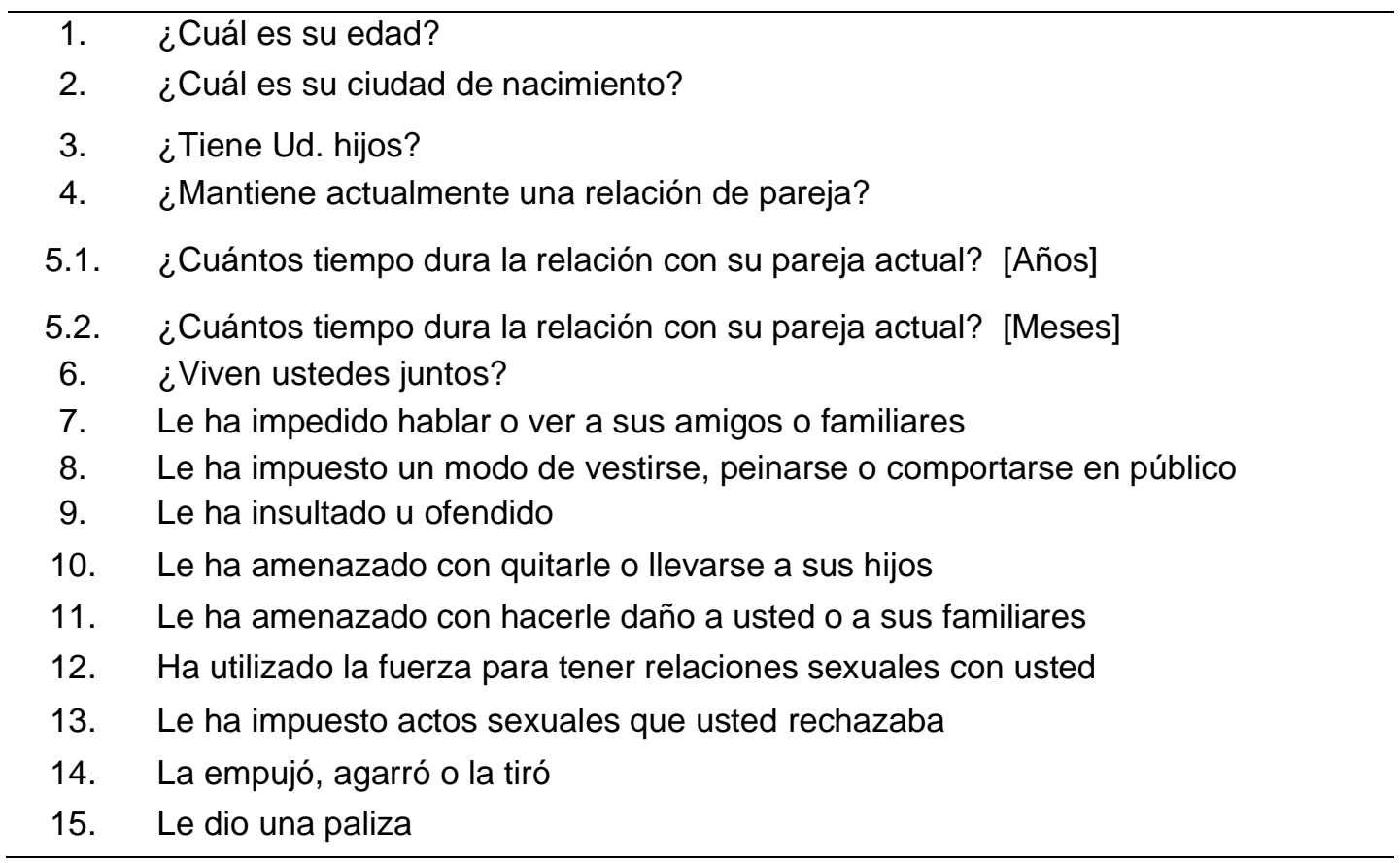

Fuente: Elaboración propia

Para las encuestas de las técnicas de respuesta aleatoria se mostraba instrucciones que las encuestadas debían seguir antes de contestar las preguntas sensibles, como se detalla a continuación con una variación para la técnica RN cambia en el punto 5.

Instrucciones: Con la aplicación para smartphone: “Generador aleatorio" seguimos las siguientes instrucciones:

1. Elegir la opción número aleatorio.

2. Definir el número mínimo y el número máximo (en nuestro caso del 1 al 12).

1. De acuerdo con la cantidad de preguntas sensibles generamos los números aleatorios, en cantidad de resultados poner 9, que son el número de preguntas sensibles.

2. Dar clic en "Resultado aleatorio".

3. Si el número se encuentra entre el 1 y el 5, se marca la opción correspondiente a ese número; si sale un número entre el 6 y el 12, se responde a la pregunta de manera sincera.

\section{Técnica de respuesta no relacionada:}

Muestra 1

1.5. Si sale un número entre el 1 y el 5, responde a la pregunta $\mathrm{P} 2$; si sale un número entre el 6 y el 12, responde a la pregunta P1. 


\section{Muestra 2}

1.5. Si sale un número entre el 1 y el 6 , responde a la pregunta $\mathrm{P} 2$; si sale un número entre el 7 y el 15, responde a la pregunta P1.

El procedimiento aleatorio que se eligió es una aplicación de smartphone, en la que se determinó 12 números aleatorios, agrupándoles en dos grupos (1-5) y (6-12), presentadas en la tabla 2:

Tabla 2. Números aleatorios

\begin{tabular}{|c|c|c|c|c|}
\hline & \multirow{2}{*}{$\begin{array}{l}\text { Número } \\
\text { aleatorio }\end{array}$} & \multirow[t]{2}{*}{ Probabilidad } & \multicolumn{2}{|c|}{$\begin{array}{l}\text { Probabilidad } \\
\text { pregunta no } \\
\text { relacionada }\end{array}$} \\
\hline & & & $\begin{array}{c}\text { Muestra } \\
1\end{array}$ & $\begin{array}{c}\text { Muestra } \\
2\end{array}$ \\
\hline Responde a la Pregunta sensible & $6-12$ & $7 / 12$ & $7 / 12$ & $9 / 15$ \\
\hline $\begin{array}{l}\text { Marca la opción correspondiente al } \\
\text { número }\end{array}$ & $1-5$ & $5 / 12$ & $5 / 12$ & $6 / 15$ \\
\hline
\end{tabular}

En la técnica de respuesta no relacionada se colocará a la par del cuestionario de preguntas sensibles, se muestra en la siguiente tabla:

Tabla 3. Encuesta técnica respuesta no relacionada (Pregunta sensible P1-Pregunta no relacionada $\mathrm{P} 2$ )

\begin{tabular}{ccl}
\hline Pregunta & Tipo & \multicolumn{1}{c}{ Pregunta } \\
\hline $\mathbf{7}$ & P1 & Su pareja le ha impedido hablar o ver a sus amigos o familiares \\
\cline { 2 - 3 } & P2 & Ha ido al cine \\
& P1 & $\begin{array}{l}\text { Su pareja le ha impuesto un modo de vestirse, peinarse o comportarse en } \\
\text { público }\end{array}$ \\
\cline { 2 - 3 } & P2 & Ha ido al teatro \\
\hline \multirow{2}{*}{10} & P1 & Su pareja le ha insultado u ofendido \\
\cline { 2 - 3 } & P2 & Ha ido al fútbol \\
\cline { 2 - 3 } & P1 & Su pareja le ha amenazado con quitarle o llevarse a sus hijos \\
\hline 11 & P1 & Su ido a comprareja le ha amenazado con hacerle daño a usted o a sus familiares \\
\cline { 2 - 3 } & P2 & ¿Cuántas veces ha utilizado el transporte público? \\
\hline 12 & P1 & Su pareja ha utilizado la fuerza para tener relaciones sexuales con usted \\
\hline \multirow{2}{*}{13} & P2 & ¿Cuántas veces ha hecho deporte? \\
\cline { 2 - 3 } & P1 & Su pareja le ha impuesto actos sexuales que usted rechazaba \\
\hline \multirow{2}{*}{14} & P2 & ¿Cuántas veces ha ido a comprar marisco? \\
\cline { 2 - 3 } & P1 & Su pareja la empujó, agarró o la tiró \\
\hline 15 & P1 & ¿Cuántas veces ha escuchado la radio? \\
\cline { 2 - 3 } & P2 & ¿Cuántas veces ha leído el periódico? \\
\hline
\end{tabular}

Fuente: Ortega., 2017

\section{Resultados.}

Uno de los objetivos de esta investigación es comprobar la eficacia de la técnica de respuesta aleatorizada, por medio del contraste de estimaciones obtenidas con la técnica 
RD vs RA y RD vs.RN. A continuación, en las siguientes tablas se muestran los resultados de las técnicas de manera simultánea.

Tabla 3.Comparación técnica RD vs RA.

\begin{tabular}{|c|c|c|c|c|c|c|c|c|}
\hline \multirow[b]{2}{*}{ Pregunta } & \multirow[b]{2}{*}{ 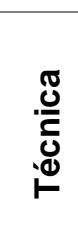 } & \multirow[b]{2}{*}{ 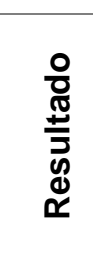 } & \multicolumn{6}{|c|}{ Categoría } \\
\hline & & & 1)Nunca & $\begin{array}{l}\text { 2)Rara } \\
\text { vez }\end{array}$ & $\begin{array}{c}\text { 3) } \\
\text { Algunas } \\
\text { veces }\end{array}$ & $\begin{array}{l}\text { 4)Mt } \\
\mathrm{s} \text { ve }\end{array}$ & $\begin{array}{l}\text { cha } \\
\text { ces }\end{array}$ & $\begin{array}{c}\text { 5) } \\
\text { Constantemente } \\
\text { siempre, } \\
\text { sistemáticament } \\
\text { e }\end{array}$ \\
\hline \multirow{6}{*}{$\begin{array}{l}\text { 7. Le ha } \\
\text { impedido } \\
\text { hablar o ver } \\
\text { a sus } \\
\text { amigos o } \\
\text { familiares }\end{array}$} & \multirow[t]{3}{*}{ RD } & Est. & 0,4799 & 0,1846 & 0,1443 & \multirow{2}{*}{\multicolumn{2}{|c|}{$\begin{array}{l}0,1107 \\
0,0751\end{array}$}} & 0,0805 \\
\hline & & (I.I) & $\begin{array}{c}0,4231 \\
\left({ }^{*}\right)\end{array}$ & 0,1405 & 0,1044 & & & 0,0496 \\
\hline & & (I.S & $\begin{array}{c}0,5366 \\
\left(*^{*}\right)\end{array}$ & 0,2286 & 0,1842 & \multicolumn{2}{|c|}{0,1464} & 0,1114 \\
\hline & \multirow[t]{3}{*}{ RA } & Est. & 0,6261 & 0,1657 & 0,1069 & \multicolumn{2}{|c|}{0,0824} & 0,0188 \\
\hline & & (I.I) & $\begin{array}{c}0,5368 \\
\left({ }^{*}\right)\end{array}$ & 0,0967 & 0,0436 & \multicolumn{2}{|c|}{0,0218} & 0 \\
\hline & & (I.S & $\begin{array}{c}0,7154 \\
\left({ }^{*}\right)\end{array}$ & 0,2347 & 0,1703 & \multicolumn{2}{|c|}{0,1431} & 0,0713 \\
\hline \multirow{6}{*}{$\begin{array}{l}8 . \quad \text { Le ha } \\
\text { impuesto } \\
\text { un modo de } \\
\text { vestirse, } \\
\text { peinarse o } \\
\text { comportars } \\
\text { e en público } \\
\left({ }^{\star *}\right)\end{array}$} & \multirow[t]{3}{*}{ RD } & Est. & 0,47651 & $\begin{array}{c}0,2080 \\
5\end{array}$ & 0,10403 & \multicolumn{2}{|c|}{0,12416} & 0,08725 \\
\hline & & (I.I) & 0,4198 & 0,162 & 0,0694 & \multirow{2}{*}{\multicolumn{2}{|c|}{$\begin{array}{l}0,0867 \\
0,1616\end{array}$}} & 0,0552 \\
\hline & & (I.S & 0,5332 & 0,2541 & 0,1387 & & & 0,1193 \\
\hline & \multirow[t]{3}{*}{ RA } & Est. & 0,66531 & $\begin{array}{c}0,1363 \\
3\end{array}$ & 0,02857 & \multicolumn{2}{|c|}{0,05306} & 0,11673 \\
\hline & & (I.I) & 0,5757 & 0,07 & 0 & \multicolumn{2}{|c|}{0} & 0,0524 \\
\hline & & $\stackrel{(I . S}{)}$ & 0,755 & 0,2026 & 0,0825 & \multicolumn{2}{|c|}{0,1102} & 0,1811 \\
\hline \multirow{6}{*}{$\begin{array}{l}\text { 9. Le ha } \\
\text { insultado u } \\
\text { ofendido } \\
\left({ }^{\star *}\right)\end{array}$} & RD & Est. & 0,4664 & 0,255 & 0,1409 & \multicolumn{2}{|c|}{0,1141} & 0,0235 \\
\hline & & (I.I) & 0,4098 & 0,2055 & 0,1014 & \multirow{2}{*}{\multicolumn{2}{|c|}{$\begin{array}{c}0,078 \\
0,1502\end{array}$}} & 0,0063 \\
\hline & & $\stackrel{(I . S}{)}$ & 0,5231 & 0,3045 & 0,1804 & & & 0,0407 \\
\hline & RA & Est. & 0,5233 & 0,2539 & 0,1216 & \multicolumn{2}{|c|}{0,0531} & 0,0482 \\
\hline & & (I.I) & 0,4357 & 0,1781 & 0,0568 & \multicolumn{2}{|c|}{0} & 0 \\
\hline & & (I.S & 0,6108 & 0,3296 & 0,1865 & \multicolumn{2}{|c|}{0,1102} & 0,1047 \\
\hline \multirow{6}{*}{$\begin{array}{l}\text { 10. Le ha } \\
\text { amenazado } \\
\text { con quitarle } \\
\text { o llevarse a } \\
\text { sus hijos }\end{array}$} & RD & Est. & 0,8356 & 0,0738 & 0,0302 & \multicolumn{2}{|c|}{0,0336} & 0,0268 \\
\hline & & (I.I) & $0,7935\left(^{*}\right)$ & 0,0441 & 0,0108 & \multirow{2}{*}{\multicolumn{2}{|c|}{$\begin{array}{c}0,0131 \\
0,054\end{array}$}} & $0,0085\left(^{*}\right)$ \\
\hline & & $\stackrel{(I . S}{)}$ & $0,8777\left(^{*}\right)$ & 0,1035 & 0,0496 & & & $0,0452\left(^{*}\right)$ \\
\hline & RA & Est. & 1 & 0,0041 & 0 & 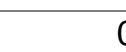 & & 0 \\
\hline & & (I.I) & $1\left(^{*}\right)$ & 0 & 0 & C & & $0\left(^{*}\right)$ \\
\hline & & $\stackrel{(I . S}{)}$ & $1\left(^{*}\right)$ & 0,0544 & 0,043 & 0,0 & & $0\left(^{*}\right)$ \\
\hline & & & & & Cate & goría & & \\
\hline Pregunta & 胥 & 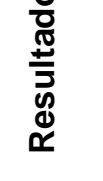 & $\begin{array}{c}\text { 1) } \\
\text { Nunca }\end{array}$ & $\begin{array}{l}\text { 2) Una } \\
\text { vez }\end{array}$ & $\begin{array}{l}\text { 3) } 2-3 \\
\text { veces }\end{array}$ & $\begin{array}{l}\text { 4) } 4-10 \\
\text { veces }\end{array}$ & $\begin{array}{c}5) \\
\text { Más } \\
\text { de } 10 \\
\text { veces }\end{array}$ & $\begin{array}{c}6) \\
\text { Prácticament } \\
\text { e todos los } \\
\text { días/siempre }\end{array}$ \\
\hline $\begin{array}{l}\text { 11. Le } \\
\text { ha }\end{array}$ & $\begin{array}{l}\mathbf{R} \\
\mathbf{D}\end{array}$ & Est. & 0,7349 & 0,2483 & $\begin{array}{c}0,016 \\
8\end{array}$ & 0 & 0 & 0 \\
\hline $\begin{array}{l}\text { amenazad } \\
\text { o con }\end{array}$ & & (I.I) & $\begin{array}{c}0,6848\left(^{*}\right. \\
\text { ( }\end{array}$ & $0,1993\left(^{*}\right)$ & $\begin{array}{c}0,002 \\
2\end{array}$ & 0 & 0 & 0 \\
\hline $\begin{array}{l}\text { hacerle } \\
\text { daño a }\end{array}$ & & (I.S) & $0,785\left(^{*}\right)$ & $0,2974\left(^{*}\right)$ & $\begin{array}{c}0,031 \\
4\end{array}$ & 0 & 0 & 0 \\
\hline
\end{tabular}




\begin{tabular}{|c|c|c|c|c|c|c|c|c|}
\hline \multirow{3}{*}{$\begin{array}{l}\text { usted o a } \\
\text { sus } \\
\text { familiares }\end{array}$} & \multirow{3}{*}{$\begin{array}{l}\mathbf{R} \\
\mathbf{A}\end{array}$} & Est. & 0,5624 & 0,0678 & $\begin{array}{c}0,097 \\
1\end{array}$ & 0 & $\begin{array}{c}0,033 \\
5\end{array}$ & 0,24 \\
\hline & & (I.I) & $\begin{array}{c}0,4667 \text { ( }^{*} \\
\text { ) }\end{array}$ & $0,0039\left(^{*}\right)$ & $\begin{array}{c}0,029 \\
6\end{array}$ & 0 & 0 & 0,1725 \\
\hline & & (I.S) & $\begin{array}{c}0,6582\left({ }^{*}\right. \\
\text { ) }\end{array}$ & $0,1316\left(^{*}\right)$ & $\begin{array}{c}0,164 \\
7\end{array}$ & 0,0528 & $\begin{array}{c}0,092 \\
6\end{array}$ & 0,3075 \\
\hline \multirow{6}{*}{$\begin{array}{l}\text { 12. Ha } \\
\text { utilizado la } \\
\text { fuerza para } \\
\text { tener } \\
\text { relaciones } \\
\text { sexuales } \\
\text { con usted }\end{array}$} & \multirow[t]{3}{*}{$\begin{array}{l}\text { R } \\
\text { D }\end{array}$} & Est. & 0,698 & 0,2617 & $\begin{array}{c}0,036 \\
9\end{array}$ & 0 & $\begin{array}{c}0,003 \\
4\end{array}$ & 0 \\
\hline & & (I.I) & $\begin{array}{c}0,6459{ }^{*} \\
\text { * }\end{array}$ & 0,2118 & $\begin{array}{c}0,015 \\
5\end{array}$ & 0 & 0 & 0 \\
\hline & & (I.S) & $\begin{array}{c}0,7501{ }^{*} \\
\text { ( }\end{array}$ & 0,3117 & $\begin{array}{c}0,058 \\
3\end{array}$ & 0 & $\begin{array}{c}0,009 \\
9\end{array}$ & 0 \\
\hline & \multirow{3}{*}{$\begin{array}{l}\mathbf{R} \\
\mathbf{A}\end{array}$} & Est. & 0,4694 & 0,1706 & $\begin{array}{c}0,170 \\
6\end{array}$ & 0,0188 & $\begin{array}{c}0,038 \\
4\end{array}$ & 0,1322 \\
\hline & & (I.I) & $\begin{array}{c}0,3762\left(^{*}\right. \\
\text { * }\end{array}$ & 0,0954 & $\begin{array}{c}0,095 \\
4\end{array}$ & 0 & 0 & 0,0803 \\
\hline & & (I.S) & $\underset{\text { ) }}{0,5626(*}$ & 0,2458 & $\begin{array}{c}0,245 \\
8\end{array}$ & 0,0756 & $\begin{array}{c}0,098 \\
2\end{array}$ & 0,1842 \\
\hline \multirow{6}{*}{$\begin{array}{l}\text { 13. Le ha } \\
\text { impuesto } \\
\text { actos } \\
\text { sexuales } \\
\text { que usted } \\
\text { rechazaba. } \\
\left({ }^{* *}\right)\end{array}$} & \multirow[t]{3}{*}{$\begin{array}{l}\text { R } \\
\mathbf{D}\end{array}$} & Est. & 0,6342 & 0,2047 & $\begin{array}{c}0,147 \\
7\end{array}$ & 0,0034 & 0 & 0,0101 \\
\hline & & (I.I) & 0,5795 & 0,1589 & $\begin{array}{c}0,107 \\
4\end{array}$ & 0 & 0 & 0 \\
\hline & & (I.S) & 0,6889 & 0,2505 & $\begin{array}{c}0,187 \\
9\end{array}$ & 0,0099 & 0 & 0,0214 \\
\hline & \multirow[t]{3}{*}{$\begin{array}{l}\mathbf{R} \\
\mathrm{A}\end{array}$} & Est. & 0,5429 & 0,2196 & $\begin{array}{c}0,190 \\
2\end{array}$ & 0,0188 & 0 & 0,0685 \\
\hline & & (I.I) & 0,4475 & 0,1401 & $\begin{array}{c}0,113 \\
2\end{array}$ & 0 & 0 & 0,0304 \\
\hline & & (I.S) & 0,6382 & 0,299 & $\begin{array}{c}0,267 \\
2 \\
\end{array}$ & 0,0756 & $\begin{array}{c}0,006 \\
2\end{array}$ & 0,1067 \\
\hline \multirow{6}{*}{$\begin{array}{l}\text { 14. La } \\
\text { empujó, } \\
\text { agarró o la } \\
\text { tiró }\left({ }^{* \star}\right)\end{array}$} & \multirow{3}{*}{$\begin{array}{l}\mathbf{R} \\
\mathbf{D}\end{array}$} & Est. & 0,5671 & 0,2617 & $\begin{array}{c}0,144 \\
3\end{array}$ & 0,0235 & $\begin{array}{c}0,003 \\
4\end{array}$ & 0 \\
\hline & & (I.I) & 0,5109 & 0,2118 & $\begin{array}{c}0,104 \\
4\end{array}$ & 0,0063 & 0 & 0 \\
\hline & & (I.S) & 0,6234 & 0,3117 & $\begin{array}{c}0,184 \\
2\end{array}$ & 0,0407 & $\begin{array}{c}0,009 \\
9\end{array}$ & 0 \\
\hline & \multirow[t]{3}{*}{$\begin{array}{l}\mathbf{R} \\
\mathbf{A}\end{array}$} & $E$ & 0,4204 & 0,2 & $\begin{array}{c}0,136 \\
3\end{array}$ & 0,0531 & $\begin{array}{c}0,043 \\
3\end{array}$ & 0,1469 \\
\hline & & (I.I) & 0,329 & 0,1222 & $\begin{array}{c}0,064 \\
5\end{array}$ & 0 & 0 & 0,0925 \\
\hline & & (I.S) & 0,5118 & 0,2778 & $\begin{array}{c}0,208 \\
2\end{array}$ & 0,115 & $\begin{array}{c}0,103 \\
8\end{array}$ & 0,2014 \\
\hline \multirow{6}{*}{$\begin{array}{l}\text { 15. Le dio } \\
\text { una paliza } \\
\left({ }^{* *}\right)\end{array}$} & \multirow[t]{3}{*}{$\begin{array}{l}\mathbf{R} \\
\mathbf{D}\end{array}$} & Est. & 0,6879 & 0,1846 & $\begin{array}{c}0,124 \\
2\end{array}$ & 0 & $\begin{array}{c}0,003 \\
4\end{array}$ & 0 \\
\hline & & (I.I) & 0,6353 & 0,1405 & $\begin{array}{c}0,086 \\
7\end{array}$ & 0 & 0 & 0 \\
\hline & & (I.S) & 0,7405 & 0,2286 & $\begin{array}{c}0,161 \\
6\end{array}$ & 0 & $\begin{array}{c}0,009 \\
9\end{array}$ & 0 \\
\hline & \multirow[t]{3}{*}{$\begin{array}{l}R \\
A\end{array}$} & E & 0,4253 & 0,2392 & $\begin{array}{c}0,195 \\
1\end{array}$ & 0,009 & $\begin{array}{c}0,004 \\
1\end{array}$ & 0,1273 \\
\hline & & (I.I) & 0,3337 & 0,1582 & $\begin{array}{c}0,117 \\
7\end{array}$ & 0 & 0 & 0,0763 \\
\hline & & (I.S) & 0,5169 & 0,3202 & $\begin{array}{c}0,272 \\
5\end{array}$ & 0,0643 & $\begin{array}{c}0,058 \\
5\end{array}$ & 0,1784 \\
\hline
\end{tabular}

Fuente: Elaboración propia.

En la tabla 4, se puede observar que no existe solapamiento en los intervalos de confianza en los ítems en las cuales las categorías presentan un asterisco, ejemplificando respecto 
al ítem: "Le ha impedido hablar o ver a sus amigos o familiares" en la categoría nunca existen diferencias significativas entre la RD y RA, con una confianza del 95\%. Por el contrario, las preguntas $8,9,13,14$ y 15 muestran intervalos de confianza que se solapan, es decir, no existe diferencias significativas entre la RD y RA. Las respuestas mediante la técnica de respuesta no relacionada son superiores que las obtenidas mediante la RD, esto se traduce a que la población objetivo no admite su participación en ciertas situaciones etiquetadas por la sociedad.

Tabla 4. Comparación de RD vs RN

\begin{tabular}{|c|c|c|c|c|c|}
\hline Preguntas & Técnica & Media & Varianza & $\begin{array}{c}\text { Intervalo } \\
\text { de } \\
\text { confianza } \\
\text { inferior }\end{array}$ & $\begin{array}{c}\text { Intervalo } \\
\text { de } \\
\text { confianza } \\
\text { superior }\end{array}$ \\
\hline \multirow{2}{*}{$\begin{array}{l}\text { 7. Le ha impedido hablar o ver } \\
\text { a sus amigos o familiares }\end{array}$} & $\mathrm{RD}$ & 2,1275 & 1,0000 & 0,0000 & 4,3919 \\
\hline & RN & 3,3605 & 0,0587 & 2,8858 & 3,8353 \\
\hline \multirow{2}{*}{$\begin{array}{l}\text { 8. Le ha impuesto un modo de } \\
\text { vestirse, peinarse o } \\
\text { comportarse en público }\end{array}$} & $\mathrm{RD}$ & 2,1376 & 1,3600 & 0,0000 & 4,4234 \\
\hline & $\mathrm{RN}$ & 3,2861 & 0,0392 & 2,8980 & 3,6741 \\
\hline \multirow{2}{*}{ 9. Le ha insultado u ofendido } & $\mathrm{RD}$ & 1,9732 & 1,1308 & 0,0000 & 4,0575 \\
\hline & RN & 3,0245 & 0,0494 & 2,5887 & 3,4603 \\
\hline \multirow{2}{*}{$\begin{array}{l}\text { 10.Le ha amenazado con } \\
\text { quitarle o llevarse a sus hijos }\end{array}$} & $\mathrm{RD}$ & 1,3423 & 0,9010 & 0,0000 & 3,2027 \\
\hline & $\mathrm{RN}$ & 2,9163 & 0,0579 & 2,4449 & 3,3878 \\
\hline \multirow{2}{*}{$\begin{array}{l}\text { 11. Le ha amenazado con } \\
\text { hacerle daño a usted o a sus } \\
\text { familiares }\end{array}$} & $\mathrm{RD}$ & 1,2819 & 0,4866 & 0,0000 & 2,6491 \\
\hline & RN & 3,5697 & 0,0962 & 2,9618 & 4,1776 \\
\hline \multirow{2}{*}{$\begin{array}{l}\text { 12. Ha utilizado la fuerza para } \\
\text { tener relaciones sexuales con } \\
\text { usted }\end{array}$} & $\mathrm{RD}$ & 1,3490 & 0,5852 & 0,0000 & 2,8483 \\
\hline & $\mathrm{RN}$ & 3,9776 & 0,0813 & 3,4187 & 4,5364 \\
\hline \multirow{2}{*}{$\begin{array}{l}\text { 13. Le ha impuesto actos } \\
\text { sexuales que usted rechazaba }\end{array}$} & $\mathrm{RD}$ & 1,5604 & 0,8750 & 0,0000 & 3,3939 \\
\hline & RN & 3,7255 & 0,0684 & 3,2128 & 4,2382 \\
\hline \multirow{2}{*}{ 14. La empujó, agarró o la tiró } & $\mathrm{RD}$ & 1,6342 & 0,8391 & 0,0000 & 3,4296 \\
\hline & $\mathrm{RN}$ & 4,3728 & 0,0836 & 3,8060 & 4,9395 \\
\hline \multirow{2}{*}{ 15. Le dio una paliza } & $\mathrm{RD}$ & 1,4463 & 0,7332 & 0,0000 & 3,1245 \\
\hline & $\mathrm{RN}$ & 3,3330 & 0,0846 & 2,7627 & 3,9032 \\
\hline
\end{tabular}

Fuente: Elaboración propia

Las encuestas aplicadas por la técnica RN muestran un incremento considerable en la respuesta comparada con la $\mathrm{RD}$, en la tabla 4 , se observa la comparación con intervalos de confianza el ítem 11 correspondiente a la pregunta "Le ha amenazado con hacerle daño a usted o a sus familiares", los intervalos no se solapan, se concluye que existe diferencias significativas entre la técnica RN y RD, la estimación de la media por la técnica RD es de 1.2819 y 3.5697 en la técnica RN. Respecto los ítems 12 y 14 "Ha utilizado la fuerza para tener relaciones sexuales con usted", "La empujó, agarró o la tiró", respectivamente, los intervalos de confianza no se solapan respecto a la técnica RN y RD, se concluye que hay diferencia significativa entre las dos técnicas. 
En definitiva, las técnicas de respuesta aleatorizada generan mayor índice de repuesta a las preguntas sensibles que al aplicar la encuesta directa, esto se puede evidenciar al tener estimaciones altas. Respecto a la pregunta que tuvo una diferencia mayor en la estimación de la media se ha obtenido en "La empujó, agarró o la tiró", por lo que se puede decir que es una pregunta muy sensible para las mujeres encuestadas, así como también; "Ha utilizado la fuerza para tener relaciones sexuales" y "Le ha amenazado con hacerle daño a usted o a sus familiares".

\section{Conclusiones.}

- Los resultados al aplicar los métodos de respuesta aleatorizada (Liu y Chow, Respuesta no relacionada) empleados en la investigación, nos permiten comparar los mismos con la técnica directa, con la finalidad de conocer la violencia a la que se ven expuestas las estudiantes de la Facultad de Ciencias Políticas y Administrativas de la Universidad Nacional de Chimborazo, mediante la aplicación de una encuesta aplicada a una muestra representativa de la población, a un $95 \%$ de confianza.

- El recurso de respuestas por ordenador tuvo gran receptibilidad en la población predispuesta a contestar de manera veraz y sin sentirse vulnerados, ni señalados, puesto también es gracias a que las encuestas no fueron de forma física sino utilizando una aplicación de internet. Se efectuó satisfactoriamente la obtención de datos mediante la aplicación de la técnica directa y la técnica aleatoria (Liu y Chow, Greenberg) respectivamente, para estudiar características sensibles que muchas veces son consideradas como un estigma social.

- Los resultados presentados en este trabajo nos permiten conocer que las técnicas estadísticas aplicadas en cualquier campo generan uno de los mayores aportes en conocimiento de las realidades existentes de nuestra sociedad y es una de las maneras más eficaces de eliminar los límites de distancia para obtener datos de manera eficaz de los encuestados.

\section{Referencias bibliográficas.}

Abdul-Ela A., Horvitz D. and Greenberg B. (1967). A multiproportions randomized response model. (62:990-1008.).

Antonak R. and Lvneh H. (1995). Randomized response technique: a review and proposed extension to disability attitude research. (1:97-145.).

Bottalessandra, A. G. (2013). Violencia contra la mujer en América Latina y el Caribe. Washington: Organización Panamericana de la Salud.

Camacho, G. (2014). La Violencia de Género contra las mujeres en el Ecuador: Análisis de los resultados de la Encuesta Nacional sobre Relaciones Familiares y Violencia de Género contra las Mujeres. La Violencia de Género contra las mujeres en el Ecuador:. 
Censos, I. N. (2012). Encuesta Nacional de Relaciones Familiares y Violencia de Género contra las Mujeres. Obtenido de https://web.archive.org/web/20171215082657/http://www.ecuadorencifras.gob.e c/documentos/web-inec/Infografias/violenciagenero.pdf

Censos, I. N. (2019). Encuesta Nacional sobre Relaciones Familiares y Violencia de Género contra las Mujeres -ENVIGMU.

Chaudhuri A. and Mukerjee R. (1988). Randomized Response: Theory and Techniques.

Chávez, Y., \& Juárez, A. (2016). Violencia de género en Ecuador. Revista Publicando, 3(8), 104-115.

Devore J. (1977). A note on the randomized response techique. 6(1525-1529).

Fox J. and Tracy P. (1986). Randomized Response: A Method for Sensitive Surveys.

Greenberg B., Lafif A., Abul-Ela A., Walt R., Daniel G., and Horvitz D. (1969). The Unrelated Question Randomized Response Model: Theoretical Framework. 64(326).

Hidalgo N., Aguirre C., Luna I., Suasnavas A. and Albán A. (2019). Encuesta Nacional sobre Relaciones Familiares y Violencia de Género contra las mujeres (ENVIGMU), Ecuador en Cifras. Encuesta Nacional sobre Relaciones Familiares y Violencia de Género contra las mujeres .

Horvitz D., Shah B., and Simmons, W. (1967). The unrelated question RR model. VA: ASA( 65-72).

Joseph, G., Javaid, S., Andres, L., Chellaraj, G., Solotaroff, J., \& Rajan, S. (2017). Underreporting of gender-based violence in Kerala, India : an application of the list randomization method. Policy Research Working Paper(8044). Obtenido de http://americalatinagenera.org/newsite//images/cdrdocuments/publicaciones/web_nuevas_expresiones_de_criminalidad.pdf

Kim, W. D. (2005). Some New Results on the Multinomial Randomized Response Model. (34: 4).

Liu P. and Chow L. (1976). A new discrete quantitative randomized response models for quantitative data. (71:72-73).

Mangat, S. R. (1990). An alternative randomized response procedure. 77(439-442).

Moors, J. A. (1971). Optimization of the Unrelated Question Randomized Response Model. 39.

Nacional, A. (2018). Ley Orgániza Integral para Prevenir y Erradicar la Violencia contra las mujeres. Quito.

O.N.U. (20 de diciembre de 1993). Declaración sobre la eliminación de la violencia contra la mujer. Obtenido de https://www.ohchr.org/sp/professionalinterest/pages/violenceagainstwomen.aspx

Salud, O. M. (2013). Estimaciones mundiales y regionales de la violencia contra la mujer: prevalencia y efectos de la violencia conyugal y de la violencia sexual no conyugal en la salud.

Scheers N. (1992). A review of randomized response techniques. Measurement and Evaluation. (25:27-41.).

Soberanis, G. R. (2008). Muestreo de respuestas aleatorizadas en poblaciones finitas: un enfoque unificador. 42(no.5). 
Umesh U. and Peterson R. (1991). A critical evaluation of the randomized response method. (20:104-138.).

Warner, S. (1965). Randomized response: a survey technique for eliminating evasive answer bias. 60(63-69).

\section{【 Ciencia}




\section{PARA CITAR EL ARTÍCULO INDEXADO.}

Morocho Barrionuevo, C. E., Morocho Barrionuevo, T. P., \& Campoverde Santos, D. K. (2021). Diseño y análisis de la violencia de género aplicando la técnica de respuesta $\begin{array}{llll}\text { aleatoria } & \text { C ConcienciaDigital, } & 4(1.2), & 338-357 .\end{array}$ https://doi.org/10.33262/concienciadigital.v4i1.2.1602

\section{Ciencia LDigital}

El artículo que se publica es de exclusiva responsabilidad de los autores y no necesariamente reflejan el pensamiento de la Revista Conciencia Digital.

El artículo queda en propiedad de la revista y, por tanto, su publicación parcial y/o total en otro medio tiene que ser autorizado por el director de la Revista Conciencia Digital.

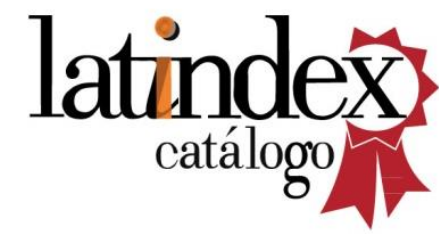

